\title{
Measurement of FRFs and modal identification in case of correlated multi-point excitation
}

\author{
U. Fuellekrug*, M. Boeswald, D. Goege and Y. Govers \\ Institute of Aeroelasticity, German Aerospace Center, DLR Goettingen, Bunsenstrasse 10, 37073 Goettingen, \\ Germany
}

Received 2007

Revised 2007

\begin{abstract}
The modal identification of large and dynamically complex structures often requires a multi-point excitation. Sine sweep excitation runs are applied when it is necessary to concentrate more energy on each line of the frequency spectrum. The conventional estimation of FRFs from multi-point excitation requires uncorrelated excitation signals. In case of multi-point (correlated) sine sweep excitation, several sweep runs with altered excitation force patterns have to be performed to estimate the FRFs. An alternative way, which offers several advantages, is to process each sine sweep run separately. The paper first describes the conventional method for FRF estimation in case of multi-point excitation, followed by two alternative methods applicable in case of correlated excitation signals. Both methods generate a virtual single-point excitation from a single run with multi-point excitation. In the first method, an arbitrary structural point is defined as a virtual driving point. This approach requires a correction of the modal masses obtained from modal analysis. The second method utilizes the equality of complex power to generate virtual FRFs along with a single virtual driving point. The computation of FRFs and the modal identification using virtual single-point excitation are explained. It is shown that the correct set of modal parameters can be identified. The application of the methods is elucidated by an illustrative analytical example. It could be shown that the separate evaluation of symmetric and anti-symmetric multi-point excitation runs yield obviously better and more reliable results compared to the conventional method. In addition, the modal analysis of the separate symmetric and anti-symmetric excitation runs is easier, since the stabilization diagrams are easier to interpret. The described methods were successfully applied during the Ground Vibration Tests on Airbus A380 and delivered excellent results. The methods are highly advantageous and may thus be established as a new standard procedure for testing aerospace structures.
\end{abstract}

Keywords: Structural dynamics, experimental modal analysis, multi-point sine sweep excitation, measurement of FRF, identification of modal masses

\section{Introduction}

In the last years a concept for testing large aerospace structures has been developed in Europe [1] and was applied for the testing of all new Airbus prototypes since 1999. The flow chart of Figure 1 surveys the modal identification concept.

For the modal identification of such large and dynamically complex aerospace structures a multiple-input excitation is often required. Within the above mentioned modal identification concept mainly sine sweep excitation runs are applied in order to concentrate more energy on each line of the frequency spectrum. When testing aircraft,

\footnotetext{
*Corresponding author. E-mail: Ulrich.Fuellekrug@dlr.de.
} 


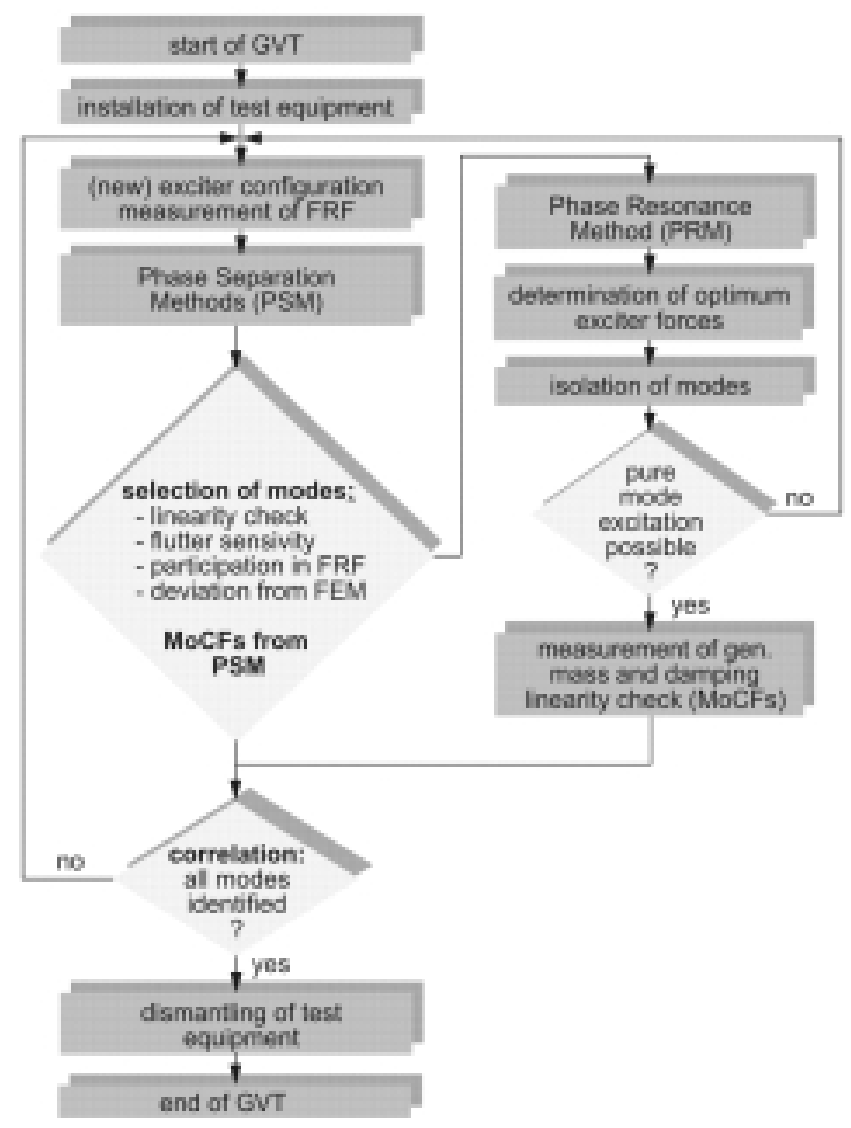

Fig. 1. Modal identification concept for large aerospace structures.

the symmetry of the tested structures suggests in many cases separate symmetric and anti-symmetric excitation runs. The symmetric and anti-symmetric excitation frequently causes the separate occurrences of symmetric and anti-symmetric modes in the measured responses. Thus, the separate dynamic responses exhibit less resonance peaks which simplifies the modal identification. However, the conventional estimation of FRFs requires uncorrelated excitation forces and, thus, in case of sine sweep excitation the simultaneous evaluation of the respective symmetric and anti-symmetric excitation runs with linearly independent excitation force vectors. The major drawbacks are that the frequency range and frequency resolution of the individual runs have to be identical, that non-linear effects occurred in one run may disperse and influence the overall results, see [2], and that the modal identification process can not be started until all runs are completed.

The general theory for FRF measurements with multi-point excitation has been well investigated in the past, see e.g. [3,4]. A newer contribution investigates multi-point FRF techniques for excitation, averaging and processing [5]. However, none of these references investigates the above described case with correlated multi-point excitation. The intention of this paper is to propose novel methods which enable the separate evaluation of symmetric and anti-symmetric excitation runs in case of correlated multi-point excitation.

\section{Basic equations and conventional FRF estimation}

In this section the basic equations are presented and the conventional FRF estimation is described. 


\subsection{Basic equations}

The dynamic response of an elastomechanical structure can be obtained by solving its equation of motion

$$
[M]\{\ddot{u}(t)\}+[C]\{\dot{u}(t)\}+[K]\{u(t)\}=\{f(t)\},
$$

Where $[M],[C]$ and $[K]$ are the physical mass, damping and stiffness matrices, $\{u(t)\}$ are the dynamic displacement responses and $\{f(t)\}$ is the vector of the excitation forces. When considering harmonic excitation forces $\{f(\omega)\}$, the dynamic response can be effectively computed in the frequency domain by using the frequency response function (FRF) matrix $[H(\omega)]$

$$
\{a(\omega)\}=[H(\omega)]\{f(\omega)\},
$$

where $\{a(\omega)\}$ are the acceleration responses, i.e. the Fourier Transform of $\{\ddot{u}(t)\}$. When neglecting the modal damping coupling, such as in case of proportional damping, Eq. (2) can be written as

$$
\{a\}=\sum_{r=1}^{n}\{\psi\}_{r} \frac{-\omega^{2}}{m_{r}\left(\omega_{r}^{2}-\omega^{2}+2 \zeta_{r} \omega_{r} \omega i\right)}\{\psi\}_{r}^{T}\{f\},
$$

where $\{\psi\}_{r}$ is the $r$-th eigenvector and $\omega_{r}, \zeta_{r}, m_{r}$ are the eigenfrequency, modal damping and modal mass of mode $r$. The FRF matrix $[H(\omega)]$ equals

$$
[H(\omega)]=\sum_{r=1}^{n} \frac{-\omega^{2}\{\psi\}_{r}\{\psi\}_{r}^{T}}{m_{r}\left(\omega_{r}^{2}-\omega^{2}+2 \zeta_{r} \omega_{r} \omega i\right)}
$$

The elements of $[H(\omega)]$ are so-called accelerances and are given by

$$
H_{j k}(\omega)=\sum_{r=1}^{n} \frac{-\omega^{2} \psi_{j r} \psi_{k r}}{m_{r}\left(\omega_{r}^{2}-\omega^{2}+2 \zeta_{r} \omega_{r} \omega i\right)} .
$$

If the structure is excited at a single point $k$ the dynamic response is

$$
\{a\}=\{H(\omega)\}_{k} f_{k}=\sum_{r=1}^{n}\{\psi\}_{r} \frac{-\omega^{2} \psi_{k r} f_{k}}{m_{r}\left(\omega_{r}^{2}-\omega^{2}+2 \zeta_{r} \omega_{r} \omega i\right)} .
$$

The estimation of the modal mass $m_{r}$ requires the FRF at the excitation point (the so-called driving point). The driving point FRF of point $k$ is according to Eq. (5)

$$
H_{k k}(\omega)=\sum_{r=1}^{n} \frac{-\omega^{2} \psi_{k r}^{2}}{m_{r}\left(\omega_{r}^{2}-\omega^{2}+2 \zeta_{r} \omega_{r} \omega i\right)} .
$$

For the modal identification from multiple-input excitation, FRFs are required as an input for the Phase Separation Techniques. Two different approaches can be used to obtain FRFs from multi-point excitation. The standard, conventional FRF estimation processes the responses of independent excitation runs and yields multiple columns of the FRF matrix, each related to unit forces at the individual driving points. As an alternative method, virtual FRFs can be computed from a single excitation run. These virtual FRFs are readily available after a single excitation run is completed. Thus the combination of the responses of different excitation runs is no longer required for the estimation of FRFs.

\subsection{Conventional method for FRF estimation}

The following equations are derived for a two-point excitation of symmetric structures such as aircraft. The use of two shakers with sine sweep excitation allows for the excitation of symmetric and anti-symmetric modes in separate sweep runs. The corresponding input-output relationship of Eq. (2) can thus be extended to include the two runs

$$
\left[\{a(\omega)\}_{S}\{a(\omega)\}_{A}\right]=[H(\omega)]\left[\{f(\omega)\}_{S}\{f(\omega)\}_{A}\right]
$$


Here, $\{a(\omega)\}_{S}$ and $\{a(\omega)\}_{A}$ are the dynamic responses due to the excitation runs with a symmetric excitation force vector $\{f(\omega)\}_{S}$ and with an anti-symmetric excitation force vector $\{f(\omega)\}_{A}$. The FRF matrix can then be calculated by inversion of the $2 \times 2$ input matrix $\left[\{f(\omega)\}_{S}\{f(\omega)\}_{A}\right]$ at each frequency $\omega$

$$
[H(\omega)]=\left[\{a(\omega)\}_{S}\{a(\omega)\}_{A}\right] \quad\left[\{f(\omega)\}_{S}\{f(\omega)\}_{A}\right]^{-1} .
$$

In case of a symmetric and anti-symmetric excitation with two exciters, the input matrix is well conditioned and the inversion causes no problems. The FRF matrix $[H(\omega)]$ obtained from Eq. (9) contains two columns with the dynamic responses related to unit forces at the two individual excitation points. Even though the excitation was chosen to excite either symmetric or anti-symmetric modes, the single columns of the FRF matrix contain contributions of both, symmetric and anti-symmetric modes. Consequently, the data evaluation may be difficult and less clear. The application of Eq. (9) requires that the frequency range and the frequency resolution of both sine sweep runs must be identical. Furthermore, it can be expected that the presence of non-linear behaviour due to different force levels during the two runs may cause inconsistent FRF data.

\section{Alternative methods for FRF estimation from multi-point excitation}

An alternative and more consistent way for the estimation of FRFs from multi-point excitation is the separate evaluation of symmetric and anti-symmetric runs by means of a virtual single driving point. In the following, it is explained how a modal identification with the virtual FRFs is enabled and how the correct modal parameters are obtained. For the sake of simplicity, the following equations are derived for two-point excitation. However, the number of excitation points does not pose a limitation to the method and it can easily be expanded to several excitation points.

\subsection{Arbitrary response point as driving point}

For an excitation at two points $k$ and $l$ the dynamic response according to Eq. (3) can be written as

$$
\{a\}=\sum_{r=1}^{n}\{\psi\}_{r} \frac{-\omega^{2}}{m_{r}\left(\omega_{r}^{2}-\omega^{2}+2 \zeta_{r} \omega_{r} \omega i\right)}\left(\psi_{k r} f_{k}+\psi_{l r} f_{l}\right),
$$

where the quantity $\psi_{k r} f_{k}+\psi_{l r} f$ is the generalized force.

With the definition of a virtual force

$$
f_{v}=\sqrt{f_{k}^{2}+f_{l}^{2}}
$$

an "alike" FRF column vector $\{H\}_{v}$ (virtual single-point excitation) can be generated by dividing the dynamic response of Eq. (10) by the virtual force of Eq. (11)

$$
\{a\} \frac{1}{f_{v}}=\{H\}_{v}=\sum_{r=1}^{n}\{\psi\}_{r} \frac{-\omega^{2}}{m_{r}\left(\omega_{r}^{2}-\omega^{2}+2 \zeta_{r} \omega_{r} \omega i\right)} \frac{\left(\psi_{k r} f_{k}+\psi_{l r} f_{l}\right)}{f_{v}} .
$$

Now, one response point $\mathrm{j}$ is selected as a virtual driving point, e.g. $j=v$. (It should be noted that any response point can be selected as a virtual driving point.) The FRF for this point $v$ can be written as

$$
a_{v} \frac{1}{f_{v}}=H_{v v}=\sum_{r=1}^{n} \frac{-\omega^{2} \psi_{v r}}{m_{r}\left(\omega_{r}^{2}-\omega^{2}+2 \zeta_{r} \omega_{r} \omega i\right)} \frac{\left(\psi_{k r} f_{k}+\psi_{l r} f_{l}\right)}{f_{v}} .
$$

For any other response point $j$ the virtual FRF can be expressed by

$$
a_{j} \frac{1}{f_{v}}=H_{j v}=\sum_{r=1}^{n} \frac{-\omega^{2} \psi_{j r}}{m_{r}\left(\omega_{r}^{2}-\omega^{2}+2 \zeta_{r} \omega_{r} \omega i\right)} \frac{\left(\psi_{k r} f_{k}+\psi_{l r} f_{l}\right)}{f_{v}} .
$$

For the modal identification the complete virtual column $\{H\}_{v}=\{a\} \frac{1}{f_{v}}$ of the FRF matrix (which includes the virtual driving point FRF $H_{v v}$ ) is used as input data to a suitable Phase Separation Technique (PST). Because 
the PST assumes that the input data are consistent with conventional FRFs defined by Eqs (4) or (7) virtual modal parameters $\omega_{r}, \zeta_{r},\{\psi\}_{r}, \tilde{m}_{r}$ are estimated. The estimated virtual parameters $\omega_{r}, \zeta_{r},\{\psi\}_{r}$ are correct, because the multiple-input appears only as a scalar factor which is the same for each virtual FRF. However, the virtual modal mass $\tilde{m}_{r}$ estimated from Eq. (13) (which the PST assumes to be consistent with Eq. (7)) is not the correct modal mass $m_{r}$. Comparing coefficients of Eqs (7) and (13) delivers the following relationship between the identified virtual modal mass $\tilde{m}_{r}$ and the correct modal mass $m_{r}$

$$
\frac{\psi_{v r}^{2}}{\tilde{m}_{r}}=\frac{\psi_{v r}}{m_{r}} \frac{\left(\psi_{k r} f_{k}+\psi_{l r} f_{l}\right)}{f_{v}} .
$$

The same comparison of Eq. (5)and (14) for an arbitrary point $j$ delivers

$$
\frac{\psi_{j r} \psi_{v r}}{\tilde{m}_{r}}=\frac{\psi_{j r}}{m_{r}} \frac{\left(\psi_{k r} f_{k}+\psi_{l r} f_{l}\right)}{f_{v}} .
$$

From both these equations a correction factor for the virtual modal mass can be derived which can be used to obtain the correct modal mass from the identified virtual modal mass

$$
m_{r}=\tilde{m}_{r} \frac{\left(\psi_{k r} f_{k}+\psi_{l r} f_{l}\right)}{\psi_{v r} f_{v}}
$$

With the correction of the modal mass according to Eq. (17) all modal parameters are correctly identified. It is thus not required for the modal identification to use FRFs that are related to unity forces at single points. However, it should be noted that the Phase Separation Technique may initially deliver purely imaginary mode shapes as a result of processing virtual FRFs instead of conventional ones. The selection of an arbitrary point as driving point may cause that the quadratic term of Eq. (7) appears negative in Eq. (13). However, the correct modal mass can be computed in spite of that: A rescaling to real modes (by setting e.g. the maximum mode shape component to unity) results in an interim negative virtual modal mass $\tilde{m}_{r}$. The following correction of the virtual modal mass according to Eq. (17) delivers then the correct modal mass $m_{r}$. It can be seen that the correction of the virtual modal masses after modal analysis is required to obtain correctly scaled mode shapes.

Here, the equations and relations are derived for systems without modal damping coupling (i.e. proportional damping) so that the FRFs can be expressed in terms of real normal modes. For systems with non-proportional damping the equations for the FRF are different. However, the same way of modal identification with a Phase Separation Technique can be used. The modal " $a$ " (a factor in the state space formulation which is equivalent to the modal mass in case of complex modes) can then be used for the computation of modal masses by postulating that the different FRF representations are equivalent. Also in this case, Eq. (17) can be used for the correction of the modal masses.

\subsection{Virtual driving point of equal complex power}

An alternative method for the estimation of FRFs from multiple-input excitation is achieved by computing additional FRF data for a virtual driving point which is not associated with any response point [6].

It is postulated that the complex power of the virtual input force $f_{v}$ equals the complex power of the actual input forces [6] (again, two-point excitation is used here for the sake of simplicity)

$$
f_{v} \dot{u}_{v}=f_{k} \dot{u}_{k}+f_{l} \dot{u}_{l} \text {. }
$$

In case of harmonic vibrations this equation can likewise be written for the accelerations as

$$
f_{v} \ddot{u}_{v}=f_{k} \ddot{u}_{k}+f_{l} \ddot{u}_{l} \text {. }
$$

Defining now the amplitude of the virtual force by the vector norm of the excitation force vector

$$
f_{v}=\sqrt{f_{k}^{2}+f_{l}^{2}}
$$

the virtual acceleration $a_{v}$ can be computed from Eq. (19)

$$
a_{v}=\ddot{u}_{v}=\frac{1}{f_{v}}\left(f_{k} \ddot{u}_{k}+f_{l} \ddot{u}_{l}\right) .
$$


The dynamic response to an excitation with forces $f_{k}$ and $f_{l}$ at points $k$ and $l$ is, according to Eq. (3), for point $k$

$$
a_{k}=\sum_{r=1}^{n} \frac{-\omega^{2} \psi_{k r}}{m_{r}\left(\omega_{r}^{2}-\omega^{2}+2 \zeta_{r} \omega_{r} \omega i\right)}\left(\psi_{k r} f_{k}+\psi_{l r} f_{l}\right)
$$

and for point $l$

$$
a_{l}=\sum_{r=1}^{n} \frac{-\omega^{2} \psi_{l r}}{m_{r}\left(\omega_{r}^{2}-\omega^{2}+2 \zeta_{r} \omega_{r} \omega i\right)}\left(\psi_{k r} f_{k}+\psi_{l r} f_{l}\right) .
$$

Inserting Eqs (22) and (23) into Eq. (21) delivers the virtual acceleration $a_{v}$

$$
a_{v}=\frac{1}{f_{v}} \sum_{r=1}^{n} \frac{-\omega^{2}\left(\psi_{k r} f_{k}+\psi_{l r} f_{l}\right)}{m_{r}\left(\omega_{r}^{2}-\omega^{2}+2 \zeta_{r} \omega_{r} \omega i\right)}\left(\psi_{k r} f_{k}+\psi_{l r} f_{l}\right) .
$$

The virtual acceleration $a_{v}$ is an additional acceleration response which can be appended to the vector $\{a\}$ of measured accelerations

$$
\{a\}=\left\{\begin{array}{l}
a_{1} \\
\vdots \\
a_{N_{o}} \\
a_{v}
\end{array}\right\} .
$$

As before, the dynamic response vector $\{a\}$ is now divided by the virtual force of Eq. (20) and an "alike" (virtual) FRF column vector $\{H\}_{v}$ is generated

$$
\{a\} \frac{1}{f_{v}}=\{H\}_{v}=\sum_{r=1}^{n}\{\psi\}_{r} \frac{-\omega^{2}}{m_{r}\left(\omega_{r}^{2}-\omega^{2}+2 \zeta_{r} \omega_{r} \omega i\right)} \frac{\left(\psi_{k r} f_{k}+\psi_{l r} f_{l}\right)}{f_{v}} .
$$

For the virtual driving point $v$ the FRF can be expressed according to Eq. (24)

$$
a_{v} \frac{1}{f_{v}}=H_{v v}=\sum_{r=1}^{n} \frac{-\omega^{2}}{m_{r}\left(\omega_{r}^{2}-\omega^{2}+2 \zeta_{r} \omega_{r} \omega i\right)} \frac{\left(\psi_{k r} f_{k}+\psi_{l r} f_{l}\right)^{2}}{f_{v}^{2}} .
$$

Here the last term is quadratic. The reason is the definition of the virtual driving point acceleration according to Eqs (21) and (24). For any other response point $j$ the (virtual) FRF can be expressed by

$$
a_{j} \frac{1}{f_{v}}=H_{j v}=\sum_{r=1}^{n} \frac{-\omega^{2} \psi_{j r}}{m_{r}\left(\omega_{r}^{2}-\omega^{2}+2 \zeta_{r} \omega_{r} \omega i\right)} \frac{\left(\psi_{k r} f_{k}+\psi_{l r} f_{l}\right)}{f_{v}} .
$$

For the modal identification the complete column $\{H\}_{v}=\{a\} \frac{1}{f_{v}}$ (which includes the virtual driving point FRF $H_{v v}$ ) is used as input data to a suitable Phase Separation Technique (PST). Because the PST assumes that the input data are consistent with conventional FRFs defined by Eqs (4) or (7) virtual modal parameters $\omega_{r}, \zeta_{r},\{\psi\}_{r}, \tilde{m}_{r}$ are estimated. In contrast to the previously described method, all the virtual parameters $\omega_{r}, \zeta_{r},\{\psi\}_{r}, \tilde{m}_{r}$ are identified correctly. This shall be proved in the following by considering the virtual modal mass. Comparing coefficients of Eqs (7) and (27) delivers the following relationship between the identified virtual modal mass $\tilde{m}_{r}$ and the correct modal mass $m_{r}$

$$
\frac{\psi_{v r}^{2}}{\tilde{m}_{r}}=\frac{1}{m_{r}} \frac{\left(\psi_{k r} f_{k}+\psi_{l r} f_{l}\right)^{2}}{f_{v}^{2}} .
$$

The same comparison of Eqs (5) and (28) for an arbitrary point $j$ delivers

$$
\frac{\psi_{j r} \psi_{v r}}{\tilde{m}_{r}}=\frac{\psi_{j r}}{m_{r}} \frac{\left(\psi_{k r} f_{k}+\psi_{l r} f_{l}\right)}{f_{v}} .
$$

Since both equations have to be fulfilled it follows that 


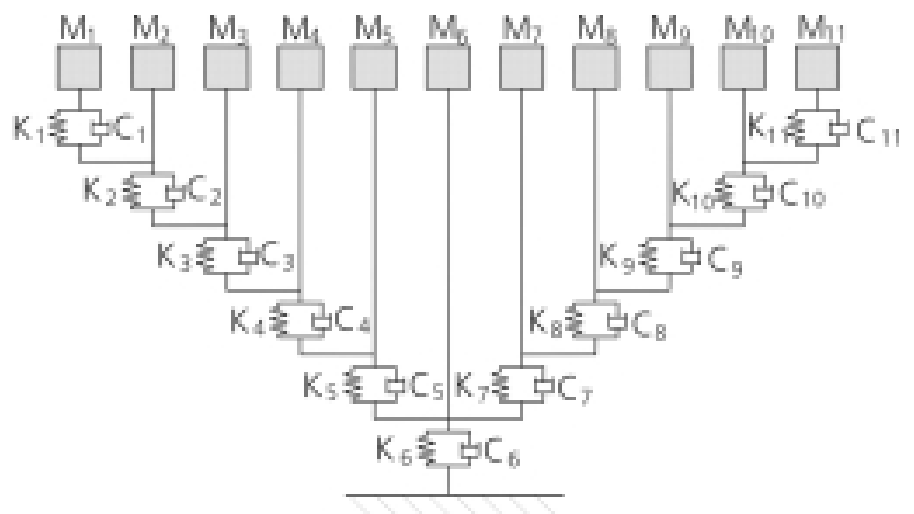

Fig. 2. Analytical vibration system with $11 \mathrm{DoF}$.

$$
\frac{\left(\psi_{k r} f_{k}+\psi_{l r} f_{l}\right)}{f_{v}}=1
$$

The validity of this equation can also be proved by a detailed derivation, which is not presented here. Equation (31) is a consequence of equal complex power of actual input and virtual input, which is postulated and defined in Eq. (18).

It can be concluded, that the identified modal mass $\tilde{m}_{r}$ is already the correct modal mass and no subsequent correction is required. However, it should be noted that the resulting mode shape vector contains an additional vector component associated with the virtual driving point. This virtual component of the mode shape vector can be removed after modal analysis and the mode shape vector can be rescaled afterwards (if necessary).

\subsection{Summary of steps for modal identification from virtual FRFs}

The steps for identifying modal parameters from virtual FRFs obtained from multi-point excitation are as follows:

- Select a suitable exciter configuration. Define forces $\{f(\omega)\}$, frequency range and sweep velocity.

- Perform a sine sweep run, measure forces $\{f(\omega)\}$ and structural responses $\{a(\omega)\}$.

- Compute a virtual force $f_{v}$ (at an arbitrary frequency if the forces are not constant) according to Eq. (11) or Eq. (20).

- Select a point as virtual point (first method) or compute a virtual acceleration $a_{v}$ according to Eq. (21) (second method).

- For using the second method, append the virtual acceleration $a_{v}$ to the vector of measured accelerations $\{a(\omega)\}$ according to Eq. (25).

- Compute an "alike" (virtual) column vector of the FRF matrix by using Eq. (12) or Eq. (26) $\{H\}_{v}=\{a\} \frac{1}{f_{v}}$.

- Utilize the virtual FRF data $\{H\}_{v}$ (with adequate definition of response points and the virtual driving point) as an input for a suitable Phase Separation Technique.

- Identify modal parameters $\omega_{r}, \zeta_{r},\{\psi\}_{r}, \tilde{m}_{r}$ with the Phase Separation Technique from the virtual FRFs. Scale the mode shapes $\{\psi\}_{r}$ in an appropriate manner (e.g. maximum unity component) and adjust the modal mass $\tilde{m}_{r}$ according to the mode shape scaling. In case of the first method, correct the modal mass $\tilde{m}_{r}$ according to Eq. (17). In case of the second method, a correction of the modal mass $\tilde{m}_{r}$ is not necessary. Instead, the mode shape component related to the virtual driving point should be removed and the mode shapes should be rescaled afterwards.

\section{Illustrative analytical example}

As an illustrative example an analytical vibration system with 11 degrees of freedom (DoF) is utilized, see Fig. 2. The 11 masses are coupled by linear springs and dampers. For computing dynamic responses, the exact modal 

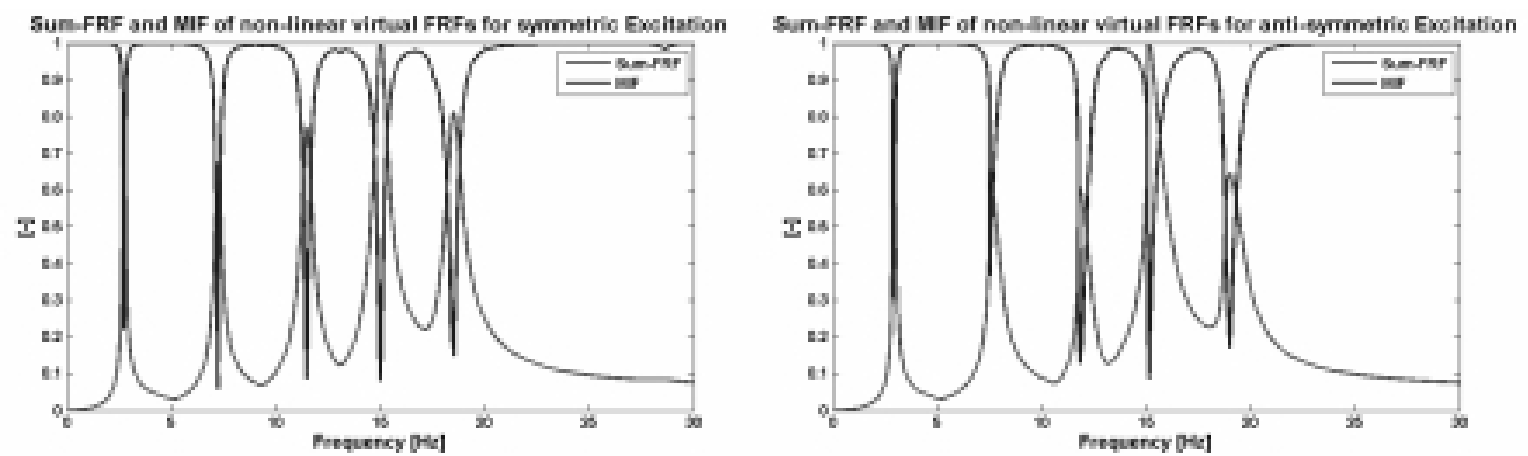

Fig. 3. Summed FRF and MIF for symmetric (left) and anti-symmetric (right) excitation runs.
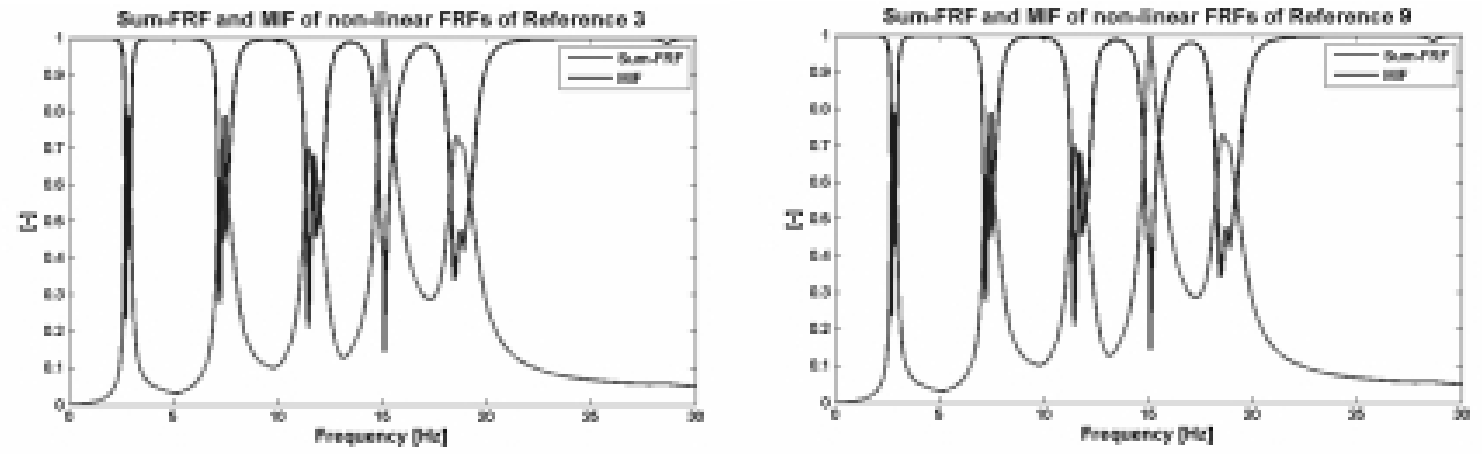

Fig. 4. Summed FRF and MIF for conventional FRF responses related to Dof \#3 (left) and DoF \#9 (right).

parameters are used and the modal damping ratio of all modes is set to $2 \%$. The vibration system exhibits 6 symmetric and 5 anti-symmetric modes with closely spaced eigenfrequencies. Excitation forces were applied at DoF \#3 and \#9. In order to introduce some non-linear behavior an additional non-linear spring element (softening cubic spring) was attached between DoF \#5 and \#7 (not shown in the figure). The attachment of the non-linear element at this position causes that only the anti-symmetric modes are affected by the non-linearity whereas the symmetric modes remain linear. By the softening characteristic of the non-linear cubic spring, the resonances of the anti-symmetric modes are shifted towards the resonances of the symmetric modes. This is expected to cause difficulties in experimental modal analysis. The non-linear frequency responses were computed by using the Harmonic Balance Response Software HBResp [7,8] (fundamental harmonic only). For the symmetric excitation, two forces of equal magnitude and both in positive direction were introduced at DoF \#3 and \#9. The anti-symmetric excitation was realized by inverting the direction of the force at Dof \#3.

The FRFs were computed with the method of Section 3.2 (virtual driving point of equal complex power). However, also the method of Section 3.1 (arbitrary response point as driving point) delivers the same FRFs, except the fact that the method of Section 3.2 delivers one additional FRF of the virtual driving point. Figure 3 shows the summed FRF (Sum-FRF) and the corresponding Mode Indicator Function (MIF) of the non-linear virtual FRFs obtained from the simulated symmetric and anti-symmetric excitation runs. It can be seen that the non-linear FRF of the symmetric excitation are still linear, whereas the resonance peaks of the anti-symmetric case are shifted towards lower frequencies due to the softening cubic spring. Figure 4 shows the summed FRF and MIF of the first and second column of the FRF-matrix $[H(\omega)]$ (related to an unit force at DoF \#3 and \#9, respectively) obtained with the conventional method for FRF estimation. It is obvious that the summed FRF and MIF of Fig. 3 exhibits less resonance peaks than the ones of Fig. 4. This is due to the fact that a single-point excitation at either DoF \#3 or \#9 excites symmetric and anti-symmetric modes. Furthermore it can be seen that some resonances of symmetric and anti-symmetric modes can no longer be distinguished due to the peak shifts of the anti-symmetric modes. 
Table 1

Deviations of identified modal parameters with new method

\begin{tabular}{ccccc}
\hline Mode \# & $\begin{array}{c}\text { Frequency } \\
{[\%]}\end{array}$ & $\begin{array}{c}\text { Damping } \\
{[\%]}\end{array}$ & $\begin{array}{c}\text { Modal Mass } \\
{[\%]}\end{array}$ & $\begin{array}{c}\text { MAC } \\
{[\%]}\end{array}$ \\
\hline 1 & 0.1 & 0.1 & -0.2 & 100.0 \\
2 & -1.3 & 1.2 & 13.5 & 100.0 \\
3 & 0.0 & 0.0 & -6.7 & 100.0 \\
4 & -2.0 & -0.4 & 17.0 & 99.6 \\
5 & 0.0 & 0.0 & -7.0 & 100.0 \\
6 & -1.4 & -5.2 & 2.4 & 99.9 \\
7 & 0.0 & 0.0 & -0.9 & 100.0 \\
8 & -1.3 & 38.5 & 5.4 & 98.6 \\
9 & 0.0 & 0.0 & 0.3 & 100.0 \\
10 & -1.3 & 10.2 & -6.5 & 99.4 \\
\hline
\end{tabular}

Table 2

Deviations of identified modal parameters with conventional method

\begin{tabular}{ccccc}
\hline Mode \# & $\begin{array}{c}\text { Frequency } \\
{[\%]}\end{array}$ & $\begin{array}{c}\text { Damping } \\
{[\%]}\end{array}$ & $\begin{array}{c}\text { Modal Mass } \\
{[\%]}\end{array}$ & $\begin{array}{c}\text { MAC } \\
{[\%]}\end{array}$ \\
\hline 1 & -0.5 & -24.3 & 2.6 & 99.5 \\
2 & -1.0 & 22.3 & -11.3 & 99.5 \\
3 & 0.6 & -16.4 & -12.6 & 99.5 \\
4 & -2.0 & 31.9 & -17.5 & 98.4 \\
5 & 0.1 & -4.9 & -32.7 & 99.2 \\
6 & -1.2 & 18.7 & -11.8 & 99.2 \\
7 & 0.0 & -51.2 & 102.7 & 99.7 \\
8 & -2.1 & 58.1 & -35.2 & 74.4 \\
9 & 0.0 & 0.3 & -36.4 & 97.3 \\
10 & -0.8 & 45.6 & -27.8 & 99.2 \\
\hline
\end{tabular}

The FRFs were analyzed with an in-house developed LSCF-type modal analysis tool (Least-Squares Complex Frequency Domain [9,10]). Since the method of Section 3.2 (virtual driving point of equal complex power) was used, the modal mass computed with the phase separation technique needs not to be corrected. However, also the method of Section 3.1 (arbitrary response point as driving point) delivers the same results. Tables 1 and 2 list the results of the modal identification in terms of deviations from the underlying linear system for eigenfrequencies, damping ratios, and modal masses. In addition, the MAC correlation of the identified modes is shown to indicate the accuracy of the identified modes. Table 1 comprises the results of the symmetric excitation run (with identification of modes 1 , $3,5,7,9)$ and the results of the anti-symmetric excitation run (identification of modes $2,4,6,8,10$ ). The symmetric modes $(1,3,5,7,9)$ are almost perfectly identified since these modes are not affected by the non-linearity. So the Phase Separation Technique (LSCF) provides accurate results. The modal identification of the anti-symmetric run yields the "best linear fit" anti-symmetric modes $(2,4,6,8,10)$ which deviate from the underlying linear reference solution. These deviations can be traced back to the non-linearity of the system and were expected to appear. The modal identification with the conventional FRF data in Table 2, however, shows significant deviations from the underlying linear solution, even for the symmetric modes which are not affected by non-linearity. The reason for this effect is that the non-linearity of the anti-symmetric modes is dispersed also to the symmetric modes. This adverse effect is introduced in the FRF estimation by the conventional method, were the responses of the linear symmetric case and the non-linear anti-symmetric case are mixed up. It can be seen from Table 1 that especially mode \#8 shows a significant deviation.

From the results of this analytical example, it can be concluded that the separate evaluation of symmetric and anti-symmetric excitation yields obviously better and more reliable results, since the experimental modal analysis is provided with FRFs which are not corrupted by mixing up linear and non-linear responses during FRF estimation. In addition, the separate evaluation of symmetric and anti-symmetric excitation runs is easier, clearer and problems with different frequency ranges and frequency resolution are avoided. 


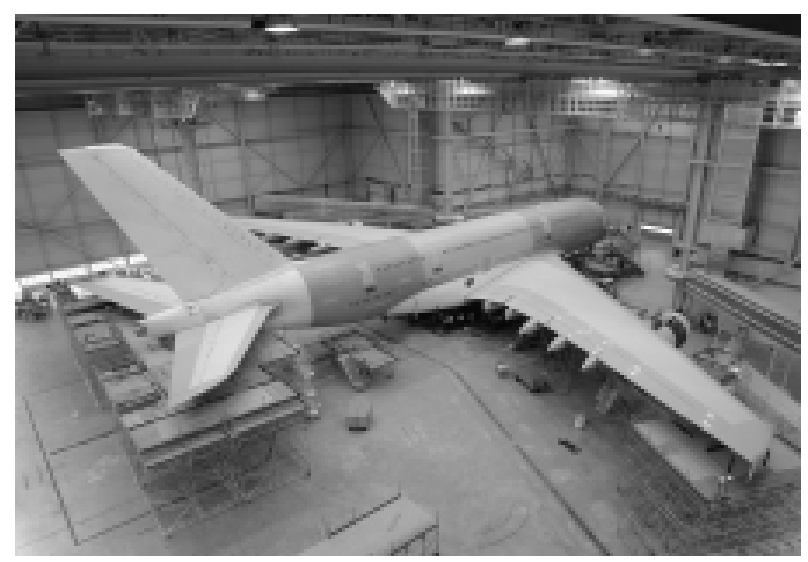

Fig. 5. Ground vibration tests on Airbus A380-800 (copyright Airbus S.A.S.).

\section{Practical application}

The proposed novel FRF estimation from correlated multi-point excitation has been successfully applied during the Ground Vibration Tests on Airbus A380-800, see [11,12]. Figure 5 shows the A380 during the Ground Vibration Tests which were performed in January 2005 by a joint test team of ONERA and DLR. The application of symmetric and anti-symmetric excitation at the wings, engines and tail plane resulted in dynamic responses which were used for the computation of FRFs. The FRFs were computed according to the method of Section 3.2 and were related to a virtual driving point as described there. The separation of symmetric and anti-symmetric excitation runs resulted in clear and manageable FRFs and simplified the modal identification process. For the evaluation of FRFs different Phase Separation Techniques (Frequency Domain Direct Parameter Estimation Identification (FDPI), Least Squares Complex Exponential (LSCE), Least Squares Complex Frequency Domain method (LSCF) and its multiple input implementation PolyMAX), which are standard tools for modal identification, were utilized [10]. A detailed description of the identified modal data is not presented here. In total, it could be concluded that the identified modal parameters appeared to be very reliable and accurate. Especially the modal masses were identified with good accuracy.

\section{Summary and conclusions}

The paper describes two methods for the estimation of FRFs in case of correlated multi-point excitation. The advantages of the methods are that the modal analysis can be started directly after the completion of a single measurement run, that the frequency ranges and frequency resolutions of the single runs need not to be identical, that non-linear effects during one run can not disperse and influence the overall results, and that the FRF evaluation is easier and much more clearer. The application of the methods requires the generation of virtual single-point excitation FRFs which are used as an input to a (conventional) Phase Separation Technique to identify the modal parameters. The application to an analytical vibration system shows that the separate modal identification for symmetric and anti-symmetric excitation runs delivers obviously better and more reliable results. In addition, the separate evaluation of symmetric and anti-symmetric excitation runs is easier and much clearer. The utilization of the methods during the Ground Vibration Tests on Airbus A380 showed that they can be used in praxis. It is expected that the described methods will also be used for evaluation of GVT data from other structures. The methods are very promising and may be established as a new standard procedure.

\section{References}

[1] G. Gloth, M. Degener, U. Fuellekrug, J. Gschwilm, M. Sinapius, P. Fargette, B. Levadoux and P. Lubrina, New Ground Vibration Testing Techniques for Large Aircraft, Sound and Vibration 35(11) (2001), 14-18. 
[2] G. Gloth and D. Goege, Handling of Non-linear Structural Characteristics in Ground Vibration Testing, Proc. of the International Conference on Noise and Vibration Engineering ISMA 2006, Leuven, Belgium, 2004, 2129-2143.

[3] R.J. Allemang, R.W. Rost and D.J. Brown, Multiple Input Estimation of Frequency Response Functions: Excitation Considerations, ASME Paper (83-DET-73) (1983)

[4] N.M.M. Maia and J.M.M. Silva, eds, Theoretical and Experimental Modal Analysis, Research Studies Press, 1997.

[5] An Overview of MIMO-FRF excitation/averaging/processing Techniques, Journal of Sound and Vibration 262 (2003), $651-675$.

[6] P. Lubrina, Ground Vibration Experiments on Large Civil Aircraft for Engine Imbalance Purpose. International Forum on Aeroelasticity and Structural Dynamics (IFASD), Amsterdam/The Netherlands, 4-6 June 2003, CEAS/AIAA/NWL/IFASD, 2003.

[7] M. Boeswald, S. Meyer and M. Link, HBResp Version 1.2 Users Guide, HBResp on the Website of the University of Kassel: http://www.unikassel.de/fb14/leichtbau/software.html.

[8] M. Boeswald, Updating of Local Non-Linear Stiffness and Damping Parameters in Large Order Finite Element Models by using Vibration Test Data, Dissertation, University of Kassel, 2005, Published as: Forschungsbericht des Deutschen Zentrums fir Luft- und Raumfahrt, ISRN DLR-FB-2005-25

[9] P. Guillaume, P. Verboven, S. Vanlanduit, H. Van der Auweraer and B. Peeters, A Poly-Reference Implementation of the Least-Squares Complex Frequency-Domain Estimator Proc. of the International Modal Analysis Conference IMAC XXI, Kissimmee, Florida, 2003.

[10] M. Boeswald, D. Goege, U. Fuellekrug and Y. Govers, A Review of Experimental Modal Analysis Methods with Respect to their Applicability to Test Data of Large Aircraft Structures, Proc. of the International Conference on Noise and Vibration Engineering ISMA 2006, Leuven, Belgium, 2006.

[11] G. Gloth and P. Lubrina, Ground Vibration Testing of the AirbusA380-800, Proc. of the International Forum on Aeroelasticity and Structural Dynamics IFASD 2005, Munich, Germany, 2005.

[12] D. Goege, M. Boeswald, U. Fuellekrug and P. Lubrina, Ground Vibration Testing of Large Aircraft-State-of-the-Art and Future Perspective, Proceedings of the $25^{t h}$ International Modal Analysis Conference (IMAC), Paper No. 258, 1-13, 2007. 

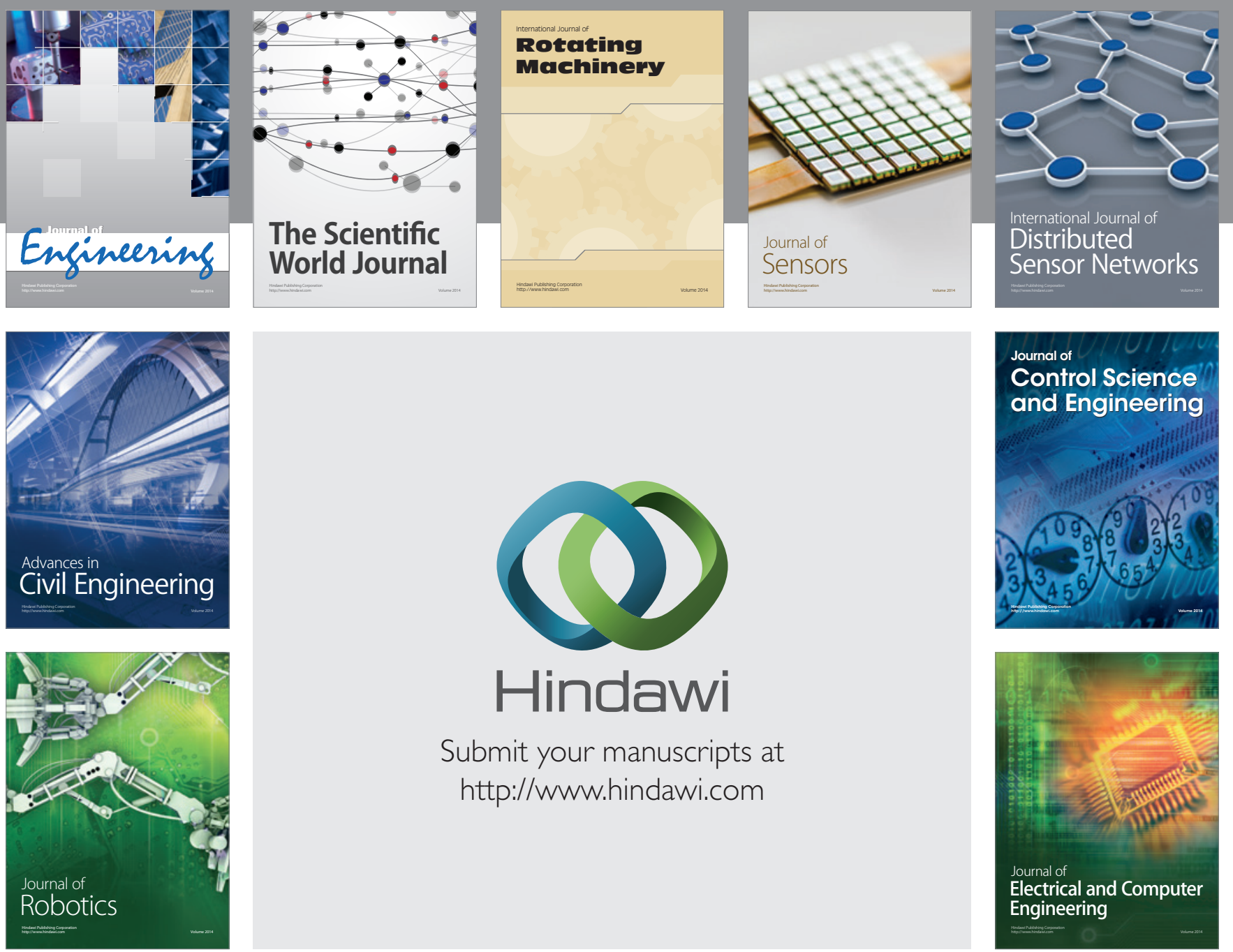

Submit your manuscripts at

http://www.hindawi.com
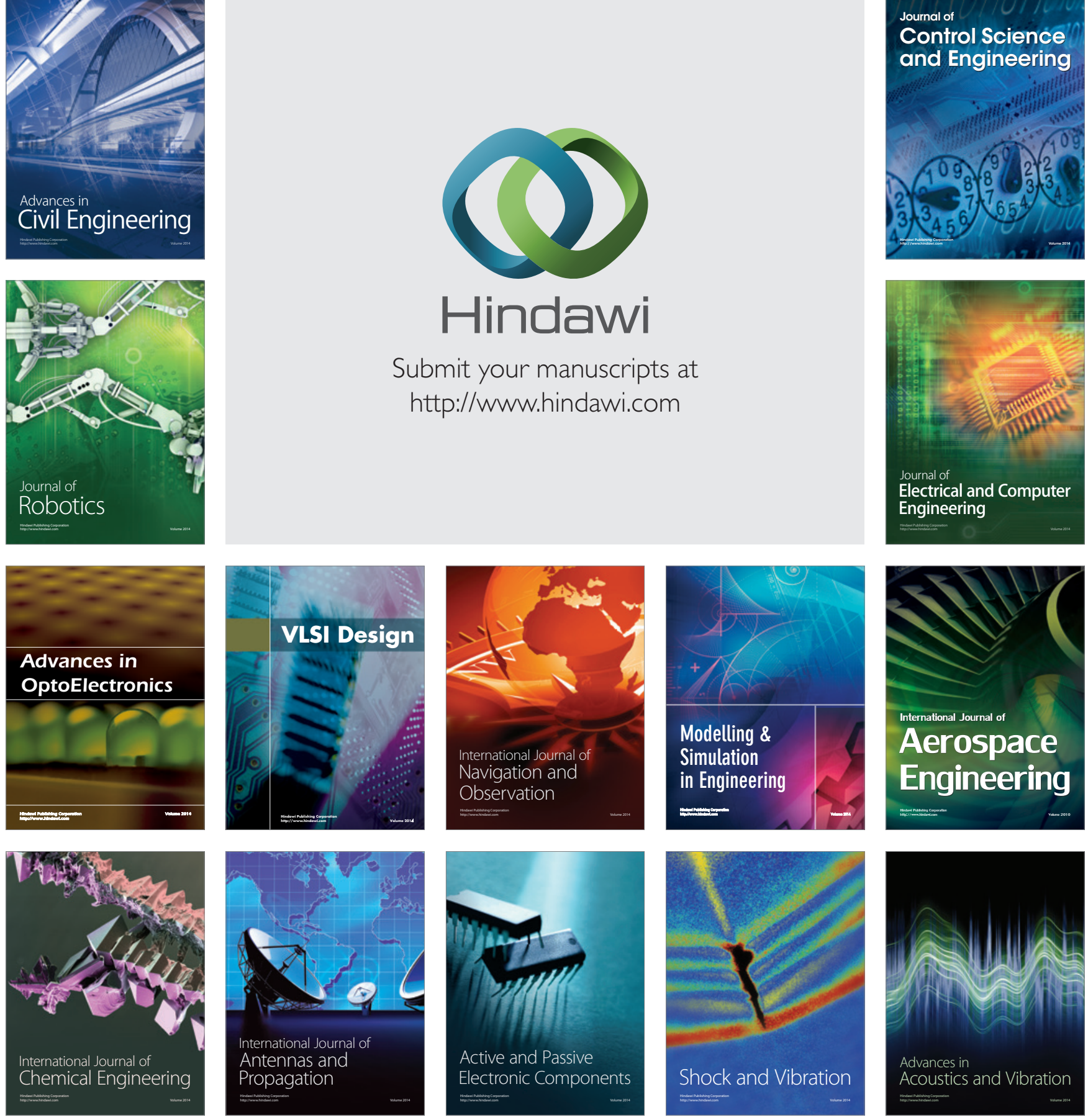